philosophical criticism of its assumptions on the other, he would be progressing out of specialism into humanism. But deliberate planning is necessary, and this is lacking. We may not altogether like the American plan, but we have no organised alternative to offer.

\section{Elementary Botany.}

(1) A Textbook of General Botany. By Gilbert M. Smith, James B. Overton, Edward M. Gilbert, Rollin H. Denniston, George S. Bryan, and Charles E. Allen. Revised edition. Pp. $x+539$. (New York: The Macmillan Co., 1928.) 16s. net. (2) A Laboratory Manual of General Botany. By Emma L. Fisk and Ruth M. Addoms. Pp. ix + 103. (New York: The Macmillan Co., 1928.) 4s. $6 d$.

(1) $\mathrm{THE}$ growing tendency towards co-operation 1 is shown by the increasing number of works that are published under joint authorship, but it is unusual to find an elementary text in which so many authors have collaborated as that before us. It is therefore with some curiosity that we peruse this experiment in multiple collaboration, for which all six authors would appear to have been collectively and severally responsible. If on one hand we are reminded of the proverbial benefits accruing from a multitude of council, on the other we recall that the broth of wisdom may suffer in homogeneity from too many compositors.

The plan of the book exhibits an especial emphasis upon the plant groups, the treatment of which occupies some 230 pages of text. By describing a larger number of types of cryptogams than are commonly exemplified in works of this standard, the reader gains a more adequate concept of the groups represented. This, however, is accomplished at the expense of the other and, from an educational point of view, probably more valuable aspects of the subject.

The families of flowering plants are treated only as part of the chapter on floral types, which contains descriptions of nineteen selected families of phanerogams. The descriptions are, however, so superficial that the reader who relied on these alone for guidance might justifiably assume that the fruits of all Ranunculaceæ were achenes, that all Cruciferous flowers possessed six stamens, and that in all Leguminosæ a single free stamen is present.

Some of the physiological matter is simply if all too briefly presented, but the explanation of the principles of diffusion of water vapour and other gases from the chlorenchyma leaves much to be desired. When we turn from the text to the figures we find even more evidence of insufficient care and thought. No subject is perhaps more commonly misunderstood by elementary students than the phenomenon of secondary thickening, which authors should therefore treat with meticulous accuracy and clarity. Here, not only is the description meagre, but also the figure which is intended to elucidate this portion of the text shows a longitudinal section of a root in which the youngest portion near the apex contains only secondary wood of the third year, whilst the second-year wood and the first-year wood are confined to the older regions ; an error that is fundamental and not one of mere inaccuracy of lettering. Again, the figure of the elm twig entirely ignores the essential feature of the branching in this species. These are merely a few of the many shortcomings that a perusal of these pages reveals.

The concept of an elementary text depends for its success not merely upon the accuracy of the in formation conveyed, or upon the amount of knowledge which it imparts, but also upon the educational aim which that knowledge serves and the subjective judgment of the authors in its selection. It would be manifestly unfair to regard the present work as evidence against the value of co-operation, but having regard to the fact that this is a "revised edition ", it is impossible not to surmise that the text would have been clearer and the errors fewer if the individual responsibility had been greater.

(2) The laboratory manual serves as a guide to the practical work which it is suggested should accompany the use of the text-book just considered. The work is divided into thirty-six exercises, of which eighteen are occupied with the study of types, five with the angiosperms, and the remaining thirteen with anatomy, morphology, and physiology. The undue emphasis upon the study of plant groups is again indicated by these proportions. The inadequacy of the anatomical work suggested is sufficiently exemplified by the treatment of secondary wood, where the only drawing which the student is asked for is a 'diagram' of a crosssection. There is no exercise in the use of microchemical tests, neither are instructions given for the use of stains and reagents for the differentiation of tissues, whilst the practical work on respiration consists of sketching a demonstration apparatus, and experiments on transpiration are ignored.

One cannot but deplore the increasing number of elementary texts that add nothing helpful either in substance or in method to botanical teaching and, at least in their own provenance, may replace texts that are real aids to the student in his difficulties.

No. 3130 , VoL. 124] 